Learning Objectives:

Introduction: Petrous temporal bone cholesteatoma (PTBC) poses a significant management challenge. The location and nature of the disease as well as surgery carry risks to vital anatomical structures with potential impact on quality of life. Traditionally an aggressive surgical approach has been used. We present our series of PTBC; their classification, management, hearing and facial nerve outcomes.

Method: A retrospective case note review was carried out for all petrous cholesteatomas managed by the senior authors from 2008-2016. The study was an analysis of service provision and therefore formal ethical approval was not required.

Results: 15 patients were included in the study (mean age $42 \mathrm{y}$ $6 \mathrm{~m}$; 10 males). Using Sanna et al.'s classification there were: 4 supralabyrinthine (Class I), 3 infralabyrinthine (II), 4 labyrinthine-apical (III), 4 massive labyrinthine (IV) and 1 apical (V). Hearing loss was a presenting symptom in $80 \%$ of patients, four of which were dead ears and $40 \%$ had a degree of facial nerve palsy. Mean follow-up was 1391 days. 5/15 patients underwent otic capsule sparing surgery. Recurrence occurred in 8 patients $(53 \%)$, who all underwent further surgery and are currently disease free. Post operatively $20 \%$ had worse hearing (all requiring a labyrinthectomy or transotic approach). Four patients had new or worse facial weakness post operatively and three of these have had subsequent facial reanimation surgery.

Conclusion: The aim in PTBC management is total exenteration of disease while minimizing complications. Compared to other series in the literature we have a higher residual/ recurrence rate due to a more conservative surgical approach in recent years. Advances in diffusion-weighted magnetic resonance imaging enable a less aggressive initial approach and directed second stage surgery in cases with residual disease.

Learning points: Long term outcomes will determine whether a less aggressive initial surgical approach is acceptable (for managing PTBC).

doi:10.1017/S002221511600133X

Free Papers (F612)

\section{ID: 612.5}

\section{A novel cartilage slicer and its performance tests}

Presenting Author: Onur Ergun

Onur Ergun, Munir Demir Bajin, Levent Sennaroglu

Hacettepe University School of Medicine

Learning Objectives:

Objective: This study investigates the performance and benefits of a novel cartilage slicer device, which we refer to as "Hacettepe cartilage slicer" for otological procedures.

Method: 41 conchal and tragal cartilage pieces were harvested using a standard surgical method from 8 fresh frozen human ears and their initial thicknesses were measured using a high accuracy digital micrometer. The harvested cartilages were then randomly sliced in 4 thickness levels using 2 different types of surgical blades. Then the thicknesses of the slices and remaining cartilages were measured. Scanning electron microscopy was utilized to determine the surface smoothness of the slices.

Results: Hacettepe cartilage slicer provided consistent results with each thickness setting and blade type. The results showed a proportional increase with the increasing thickness level with a clustering within a 0.1 milimeter distribution of the median value. The thicknesses of the slices and remaining cartilages provided evidence that our design slices the cartilage without any damage or squashing. Although both blades provided comparable satisfying results, scanning electron microscopy revealed that the slices cut with single bevel "chisel type" blade were superior regarding surface smoothness.

Conclusion: We believe that a reliable, surgical tool to slice autologous cartilage into undamaged, smooth slices at any desired thickness is major advantage for otologists. To our knowledge this study is the first to evaluate the performance of a cartilage slicer device. On the basis of this study, our novel cartilage slicer proved itself as a superior tool that is ready for human trials. A "razor sharp" disposable single bevel blade would offer the best results and slice thickness accuracy, precisely matching the desired thickness with a margin of error less than $0.1 \mathrm{~mm}$.

doi:10.1017/S0022215116001341

\section{Free Papers (F612)}

\section{ID: 612.6}

\section{Incidence, recurrence rate and prognostic} factors for cholesteatoma

\section{Presenting Author: Anders Britze}

\section{Anders Britze, Marie Møller, Therese Ovesen \\ Aarhus University Hospital}

\section{Learning Objectives:}

Purpose: The purpose of this study was to calculate long term recurrence rates of cholesteatoma and to identify significant indendepedent prognostic factors for the recurrence.

Materials and Methods: 147 children and adults operated for debuting cholesteatoma, primarily by CWU (canal wall up) mastoidectomy, at Aarhus University Hospital in the period 2001-2005 were included. Five- and ten-year Kaplan-Meier cumulative recurrence rates were calculated and significant prognostic factors were identified by Cox multivariate regression analyses. One year pre to post-operative hearing outcomes were assessed.

Results: Five and ten year recidivism rates (with confidence intervals) were $0.38(0.31-0.46)$ and $0.44(0.37-0.53)$ respectively. The same estimates from purely CWU with single-stage ossiculoplasties were $0.39(0.3-0.51)$ and 0.49 (0.39-0.60) respectively. Independent significant prognostic 
factors were: Age below 15 years, extension of cholesteatoma into the mastoid, erosion of the incus, and erosion of the stapes. Overall, there was a significant pre- to post-operative air-bone gap (ABG) improvement of $4.05 \mathrm{~dB}(1.0-7.1)$.

Conclusions: Long term recurrence rates and prognostic factors of CWU mastoidectomy help decision making in surgical approach and risk stratification of patients. Especially children with large choleateomas extending into the mastoid and with concomitant bone erosions should be carefully followed up.

doi:10.1017/S0022215116001353

\section{How do we approach cholesteatoma (N613)}

\section{ID: 613.1}

\section{Modified Bondy technique: indications and technique}

Presenting Author: Anna Lisa Giannuzzi

Anna Lisa Giannuzzi ${ }^{1}$, Enrico Piccirillo ${ }^{2}$, Mario Sanna ${ }^{2}$

${ }^{1}$ Gruppo Otologico, Piacenza, ${ }^{2}$ Gruppo Otologico

\section{Learning Objectives:}

Introduction: Modified Bondy technique in indicated in patients with epitympanic cholesteatoma, good hearing and intact pars tensa and ossicular chain. It permits to eradicate the desease with a single stage procedure. This presentation evaluates the short- $(6 \mathrm{mo})$ and long-term $(5 \mathrm{yr})$ outcomes of modified Bondy technique, with particular reference to hearing results.

Methods: Four hundred eight ears were operated on, using a modified Bondy technique between 1983 and 2013. All patients had primary acquired epitympanic cholesteatomas with intact pars tensa and intact ossicular chain in normal or goodhearing ear. Preoperative audiometric results revealed a mean air conduction pure-tone average of $27.7 \pm 9.6 \mathrm{~dB}$ (range, $10-65 \mathrm{~dB}$ ) and a mean bone conduction pure-tone average of $14.2 \pm 6.4 \mathrm{~dB}$ (range, $5-50 \mathrm{~dB}$ ). The mean preoperative airbone gap was $13.5 \pm 6.7 \mathrm{~dB}$ (range, $0-25 \mathrm{~dB}$ ). The average length of follow-up was 7.8 years (range, $5-16 \mathrm{yr}$ ).

Results: There was no recurrent cholesteatoma in the present series. A pearl-like residual cholesteatoma was found in the cavity in $7.4 \%$ of ears; $0.8 \%$ developed stenosis of meatoplasty, $1.3 \%$ exhibited retraction pockets extending to the attic. Postoperative discharging ear was observed in $3 \%$ of cases and was successfully treated with topical drops. At the long-term follow-up, the airbone gap was unchanged or improved from the preoperative level in $88 \%$ of cases. The mean postoperative short- and long-term air-bone gaps were $14.6 \pm 8.5 \mathrm{~dB}$ (range, $0-55 \mathrm{~dB}$ ) and $14.1 \pm 8.2 \mathrm{~dB}$ (range, 0-50 dB), respectively. Postoperative high-frequency sensorineural hearing loss was observed in $1.7 \%$. No dead ears were encountered postoperatively.

Conclusion: A modified Bondy operation is recommended in selected cases of epitympanic cholesteatoma in normal or good-hearing ear with an intact pars tensa and ossicular chain. Modified Bondy technique ensures complete eradication of disease while preserving a good preoperative hearing in 1-stage operation.

doi:10.1017/S0022215116001365

How do we approach cholesteatoma (N613)

\section{ID: 613.2}

How to perform a good canal wall up mastoidectomy

Presenting Author: Gianluca Piras

Gianluca Piras, Alessandra Russo, Enrico Piccirillo, Flavia D'Orazio, Sampath Chandra Prasad Rao, Mario Sanna Gruppo Otologico

Learning Objectives: How to perform a good canal wall up mastoidectomy.

Various techniques for cholesteatoma surgery have been developed, practiced, criticized, and favored by different otologists. The current dilemma regarding the choice of technique reflects differences of option between various schools of thinking in Otology. However, both the open and closed techniques have now been individualized, and the choice of procedure can be made in accordance with certain indications in order to optimize the results. In the 1960s Bill House popularized Canal Wall Up (CWU) mastoidectomies; since then CWU mastoidectomy has remained the ideal surgical treatment for pediatric cholesteatoma. This technique allows preservation of the hearing function without aestetic modification of the external ear. The limit of this technique is the incresed risk of cholesteatoma recurrence or residual; for this reason, a two-stages procedure is preferred. Indications for CWU mastoidectomy are cholesteatoma in pneumatized mastoids, children, limited epitympanic erosion, mesotympanic cholesteatoma, limited congenital cholesteatoma. Contraindications are a widespread disease, bony distruction and poor hearing. The aim of our presentation is to show technical refinements of this technique, focusing on surgical cases and results in pediatric population.

doi:10.1017/S0022215116001377

\section{How do we approach cholesteatoma (N613)}

\section{ID: 613.3}

Subtotal Petrosectomy in the management of difficult cases of cholesteatoma

\section{Presenting Author: Sampath Chandra Prasad}

Sampath Chandra Prasad, Valeria Roustan, Enrico Piccirillo, Annalisa Giannuzzi, Mario Sanna

Gruppo Otologico

Learning Objectives:

Objectives: The purpose of this study was to review the indications for subtotal petrosectomy in difficult cases of cholesteatoma, report our management of complications, as 